\title{
Philosophy of medicine 2017: reviewing the situation
}

\author{
Patrick Daly ${ }^{1}$
}

Published online: 6 November 2017

(C) Springer Science+Business Media B.V. 2017

\begin{abstract}
In this introduction to a special subsection of Theoretical Medicine and Bioethics comprising separate reviews of the Springer Handbook of the Philosophy of Medicine, The Routledge Companion to Philosophy of Medicine, and The Bloomsbury Companion to Contemporary Philosophy of Medicine, I compare the three texts with respect to their overall organization and their approach to the relation between the science and the art of medicine. I then indicate two areas that merit more explicit attention in developing a comprehensive philosophy of medicine going forward: health economics and systematic relations within the field as a whole. The reviews that follow speak for themselves.
\end{abstract}

Keywords Art of medicine $\cdot$ Health science $\cdot$ Human science $\cdot$ Medical humanism $\cdot$ Natural science $\cdot$ Philosophy of medicine

The recent publication of the three books under review in this special subsectionthe Springer Handbook of the Philosophy of Medicine, edited by Thomas Schramme and Steven Edwards [1], The Routledge Companion to Philosophy of Medicine, edited by Miriam Solomon, Jeremy Simon, and Harold Kincaid [2], and The Bloomsbury Companion to Contemporary Philosophy of Medicine, edited by James Marcum [3] - caps off a 25-year period in which contemporary philosophy of medicine has come of age as a distinct field of investigation, a period that conventionally began with Arthur Caplan's paper questioning the existence of such a field [4]. The books cover this terrain from differing perspectives and with differing objectives. The Springer Handbook offers the most comprehensive representation of the discipline, inclusive of social, metaethical, and psychiatric issues, alongside issues related to epistemology and metaphysics. The Routledge

Patrick Daly

dalypp@bc.edu

1 Lonergan Institute at Boston College, Chestnut Hill, MA, USA 
Companion achieves the most coherent success in representing the field from a naturalistic standpoint, featuring paradigmatic clinical examples in a format that is particularly suited to serve as a textbook. The editors have benefitted, I believe, in refining their pedagogical approach from prior work together on texts edited by Harold Kincaid and Jennifer McKitrick [5] and Fred Gifford [6]. The Bloomsbury Companion focuses on open questions in current research programs and thus provides a clear sense of where investigation in philosophy of medicine is headed. Marcum singles out the problematic relation between "medical scientism" and "medical humanism" as central to the coming "metaphysical turn" in philosophy of medicine, correlative to the "epistemological turn" that has characterized the past 25 years [3, pp. 22-23].

The way that one negotiates this metaphysical turn revolves around the way that one understands the reality of data of consciousness. This is critically important for philosophy of medicine because health science operates at the intersection of human science and natural science-with data of intentional consciousness paramount to the former and data of sense paramount to the latter. ${ }^{1}$ The problematic that Marcum identifies is apparent in the varied approaches to the relation between the science and the art of medicine in the three volumes under consideration. Seeking to understand medicine both as a science and as an art is an organizing principle that runs through the Springer Handbook from beginning to end, as I discuss in more detail in the book review below. Here, I focus on Kristine Bærøe's chapter on "Medicine as art and science" in order to compare the Handbook on this subject with the other two texts. Bærøe traces present day concepts of art and science to Renaissance thinkers, who distinguished humanistic disciplines concerned with understanding the meaning of the products of human activity and natural science concerned with disclosing and explaining the hidden facts of nature by experimental means. This contrast corresponds roughly to that described by C. P. Snow between the "soft discipline of human science" and the "hard science of nature" [1, p. 762], although the division is blurred by the more recent emergence of social sciences and economics that employ both hermeneutical and experimental methods. Bærøe says that the theoretical issue for philosophy of medicine and the practical issue for health policy are essentially the same: how to account for doctors' skillful integration of these disciplines-that is, general biomedical knowledge with patients' particular experiential perspectives - in the process of deciding on a course of clinical action that reasonably and considerately addresses the problem at hand. This is a complex matter and there are no simple answers. She suggests that an "allthings-considered art" - one that encompasses translation of generalized biomedical knowledge to particular circumstances, genuine moral agency, and consideration of "nonbiomedical" knowledge-might equate with a "broadly construed conception of practical, medical reasoning" [1, p. 769]. I am sympathetic to her overall treatment, but would challenge her claim that there is "no direct access to the epistemological processes that support medical practice" [1, p. 760]. What Bærøe calls nonbiomedical or tacit knowledge is verifiable in large part by reference to

\footnotetext{
1 Roger Smith presents an excellent account of the distinction between human and natural science from an Anglophone perspective in The Norton History of the Human Sciences [7].
} 
data of consciousness, which are not observable but given in experience, and can thus empirically ground such knowledge [8].

The Routledge Companion frames discussion of the science and the art of medicine in terms of the relation between narrative/humanistic data and scientific/ objective data. Danielle Spencer writes at length about the role of narrative in providing a context for understanding a patient's particular clinical situation; Ross Upshur and Benjamin Chin-Yee argue convincingly that clinical reasoning incorporates both quantitative and humanistic data when performed properly by a virtuous physician; and Alex Gamma calls into question the inadequate differentiation of the "information metaphor" [2, p. 400] in current accounts of personalized medicine, which conflate the notion of information as biological signal strength with that of information as constituted by human meaning and value. Yet the predominantly naturalistic orientation of the text is aptly reflected in Fred Gifford's endorsement of George Engel's hierarchically ordered biopsychosocial model: "[T]his view does not require endorsing holism...In particular, it doesn't require rejecting an ontological or metaphysical thesis about reductionism-that the structure of the world really is such that the higher levels are fully constituted by and can be given an explanation in terms of the lower levels" [p. 450]. Whether and how these different viewpoints can be reconciled will determine what comes of the metaphysical turn that Marcum envisions.

Those contributors to the Bloomsbury Companion who approach philosophy of medicine by asking about causation rather than hermeneutics also raise questions that bear upon the interplay between these approaches. For instance, after discussing various accounts of causal reasoning in epidemiology, Alex Broadbent closes his article by posing for future consideration a question whether epidemiology is a social or a natural science. Those contributors who work from a humanistic or hermeneutical perspective also stress the need to integrate this perspective with a natural science or evidence-based-medicine (EBM) approach. The question that looms over the whole set of essays is how these approaches are to be integrated. As noted, James Marcum speculates that this is a metaphysical issue to be worked out by developing a critical realist understanding of persons in relation to the clinical encounter. Jacob Stegenga and coauthors frame this as an epistemological problem, requiring "fine-grained analysis" of research strategies and careful articulation of the "intersection between social, ethical, and methodological aspects of medical research" [3, p. 345]. For example, they support the claim that meta-analysis should address the social context as well as the methodological details of the research in question. Michael Loughlin and coauthors understand philosophy to be an activity of critical thinking rather than a body of theory [p. 55] and favor a methodological approach to improving the status of person-centered medicine-in theory and practice-relative to EBM or other so-called objective medical models. They remark that this does not entail an algorithmic account of who is and is not a "person," valid in all circumstances, but that it does entail careful analysis of the different dimensions of the clinical encounter and medical practice in general [p. 57]. I would add that one can study the persons involved in such encounters and practice in terms of the dynamically normative operations of human inquiry and 
culture-making rather than a culture-bound set of canonical attributes [8]. But, as the authors indicate, this is work that remains to be done.

Among topics that merit more explicit attention than they receive in these compendia, I would begin with the significance of health economics for philosophy of medicine. In what ways do concerns for profit drive the engine of medical research, even or especially randomized controlled studies that meet highly valued epistemic standards? What economic and non-economic measures are appropriate for this analysis? In what way does academia underwrite an economically dominant group of actors that ranks personal gain over public well-being while touting the latter with regard to research and health policy? How does economics drive the bureaucratization of clinical practice and the regimentation of what constitutes clinical data for all practical purposes? How much does current economic thinking drive cultural malaise in the form of professional burnout and public distrust? Carl Elliott shines a light on professional demoralization and ethical compromises as the underside of the commodification of medicine [2, pp. 519-528]; Fredrik Svenæus discusses the linkage of technology to market economies and to people's worldviews concerning human finitude [3, pp. 205-226]; Keekok Lee cautions against the deliberate replacement of human capital with new technologies in health care organizations [1, pp. 661-676]; and several authors express concern about financial bias compromising the way that randomized controlled trials are conducted and reported. But this topic merits the sort of attention that EBM has received in philosophy of medicine over the past 20 years. Leah McClimans comes closest to moving philosophy of medicine in the direction I am suggesting in her discussion of the "under-theorized nature" of quality of life measures - particularly the qualityadjusted life-year (QALY) [2, pp. 335-341]. But she restricts her analysis to the epistemic utility of these measures, such as it is, much as policy makers settle on measuring economic value in terms of utility, presumably lacking a better alternative. The socioeconomic and political problems that continue to surface in the wake of technological advances in contemporary health care appear to require a different approach, in which utility ranks demonstrably lower than values like solidarity, not only ethically, as in Aristotle's ranking of different forms of friendship, but also economically, by ordering health care spending to sustainable economic growth and economic growth to sociopolitical well being [9].

Another area worth greater attention in developing a comprehensive philosophy of medicine concerns systematic understanding of what constitutes the field and its significant interrelations. As Marcum rightfully observes, current philosophy of medicine is pluralistic; and most contributors to these three texts frame their discussion in terms of discrete units of analysis or discrete subdisciplines. However, there are a few philosophers of medicine who aim for a systematic approach. In the prologue to A Philosophical Basis of Medical Practice, Edmund Pellegrino and David Thomasma write:

This book is a first step toward what we hope will become a systematic philosophy of medicine. In it, we have tried to define what we believe the nature of medicine to be, and then, what the conception means for medical practice and medical morality. Our emphasis is primarily on metaphysical, 
ontological, and moral questions. Logical and epistemological issues are touched on only tangentially. These must be aired in future if we are to move toward a comprehensive philosophy of medicine [10, p. 3].

Significantly, apropos my remarks about economics, their last chapter is titled "Medical morality and medical economics: the conflict of canons." None of the contributors to the texts under review comments on the success or failure of Pellegrino and Thomasma's overall project, although Upshur and Chin-Yee draw significantly on their account of clinical judgment [2]. Similarly, in these pages there is no discussion or appraisal of Kazem Sadegh-Zadeh's Handbook of analytic philosophy of medicine [11], which Maël Lemoine ranks as a major contribution likely to become a classic in the field [12]. How well does Sadegh-Zadeh succeed in constructing his rationalist account of medicine as a "duty-driven and normative, i.e., deontic, discipline," based on "minutely detailed logical analyses of medical language, concepts, knowledge, and decisions" and not on "sterile debate about whether medicine is a science or an art" $[11$, p. 4]? How accurate is his claim that medicine is in transition "to become an engineering science, conducted as health engineering and anthropotechnology" [p. 8]? If accurate insofar as it describes an actual historical trend, to what extent does this trend represent an intelligent development in human affairs, and to what extent does it represent a shortsighted scheme blind to the tacit schemes on which technology depends? Answering these questions will require close and explicit attention to systematic relations in health science and health care over and above the fine-grained analysis of specific topics that the editors and contributors to these volumes have so admirably advanced.

In closing, I would like to thank Mary Jean Walker and William Stempsey for the opportunity to work with them and the editors of Theoretical Medicine and Bioethics for their support in putting this set of reviews together as fitting notice of these landmark publications.

\section{References}

1. Schramme, Thomas, and Steven Edwards (eds.). 2017. Handbook of the philosophy of medicine. Dordrecht: Springer.

2. Solomon, Miriam, Jeremy R. Simon, and Harold Kincaid (eds.). 2017. The Routledge companion to philosophy of medicine. New York: Routledge.

3. Marcum, James A. (ed.). 2017. The Bloomsbury companion to contemporary philosophy of medicine. London: Bloomsbury Academic.

4. Caplan, Arthur L. 1992. Does the philosophy of medicine exist? Theoretical Medicine 13: 67-77.

5. Kincaid, Harold, and Jennifer McKitrick (eds.). 2007. Establishing medical reality: Essays in the metaphysics and epistemology of biomedical science. Dordrecht: Springer.

6. Gifford, Fred (ed.). 2011. Philosophy of medicine. Amsterdam: Elsevier.

7. Smith, Roger. 1997. The Norton history of the human sciences. New York: W. W. Norton.

8. Lonergan, Bernard. 1988. Cognitional structure. In Collection, vol. 4, ed. Collected works of Bernard Lonergan, Frederick E. Crowe, and Robert M. Doran, 205-221. Toronto: University of Toronto Press. 
9. Case, Anne, and Angus Deaton. 2015. Rising morbidity and mortality in midlife among white nonHispanic Americans in the 21st century. Proceedings of the National Academy of the Sciences of the United States of America 112: 15078-15083.

10. Pellegrino, Edmund D., and David C. Thomasma. 1981. A philosophical basis of medical practice. New York: Oxford University Press.

11. Sadegh-Zadeh, Kazem. 2015. Handbook of analytic philosophy of medicine, 2nd ed. Dordrecht: Springer.

12. Lemoine, Maël. 2012. Book review: Handbook of analytic philosophy of medicine by Kazem Sadegh-Zadeh. Revue philosophique de la France et de l'étranger 202: 556-557. 